\title{
$O$ isolamento social durante a pandemia do SARS-CoV-2 aumenta ou diminui a poluição sonora no ambiente urbano?
}

\author{
Does social isolation during the SARS-CoV-2 pandemic increase or \\ decrease noise pollution in urban environment?
}

Guilherme de Matos Abe', Laís Reis Santos', Ricardo Vieira Teles Filho'

DOI: 10.1590/0103-11042020E422

RESUMO A disseminação pandêmica do SARS-CoV-2 por mais de 100 países em todo o mundo fez com que diversos governos decretassem medidas de restrição de mobilidade social conhecidas como 'isolamento social'. Esse cenário pode ter, como efeito colateral, uma percepção de redução da poluição sonora, que pode ser corroborada pela mensuração do impacto sonoro em ambientes públicos nas cidades submetidas às medidas de isolamento social. Entretanto, há registros de crescimento de queixas às autoridades por poluição sonora advindos de zonas residenciais, o que pode indicar, contrariamente, seu aumento. Considerar essas facetas duais do assunto é importante para qualquer estudo que deseje aprofundar as análises. A poluição sonora e seus efeitos danosos sobre a saúde são de grande relevância no cenário pandêmico atual, pois podem se somar a vários outros estressores que, conjuntamente, ameaçam a saúde mental de populações neste cenário. Este trabalho, baseado em publicações científicas e de imprensa feitas durante o período, analisa e coloca em discussão as diferentes facetas dessa conjuntura que podem influenciar a elaboração de políticas públicas em saúde.

PALAVRAS-CHAVE Ruído. Infecções por coronavírus. Pandemias. Saúde mental.

\begin{abstract}
The pandemic dissemination of SARS-CoV-2 by over 100 countries worldwide caused various governments to impose social mobility restriction measures known as social isolation. This scenario may have, as a side effect, a perception of a reduction in noise pollution, which can be confirmed by measuring the sound impact in public environments in cities subjected to social isolation measures. However, there are reports of growth in complaints to the authorities for noise pollution from residential areas, which may indicate, on the contrary, its increase. Considering these dual facets of the subject is important for any study that wishes to deepen the analysis. Noise pollution and its harmful effects on health are of great relevance in the current pandemic scenario, as they can be added to several other stressors that, together, threaten the mental health of populations in such scenario. This work, based on scientific and press publications made during the period, analyzes and discusses the different facets of this situation that can influence the development of public health policies.
\end{abstract}

KEYWORDS Noise. Coronavirus infections. Pandemics. Mental health. 


\section{Introdução}

A disseminação pandêmica do SARS-CoV-2, vírus causador da Covid-19, por mais de 100 países em todo o mundo foi recebida com grande preocupação por seus governos, que passaram a adotar estratégias cada vez mais rígidas para conter tal disseminação. Uma das mais drásticas é o que se chama de 'isolamento social', 'quarentena' ou 'lockdown', que consiste em restrições de mobilidade dos cidadãos no ambiente público e de abertura de pontos comerciais, embora o grau dessas restrições varie entre os países'.

Tal conjuntura, como efeito colateral, pode promover a sensação de que 'a cidade está mais silenciosa', principalmente pela redução da circulação de pessoas e veículos em ambientes públicos. Por outro lado, o aumento do tempo no interior das residências, bem como da quantidade de pessoas nelas, pode, paradoxalmente, aumentar o registro de queixas por poluição sonora nas zonas residenciais. Assim, levanta-se a seguinte questão: as estratégias de isolamento social adotadas diante da pandemia do SARS-CoV-2 aumentam ou reduzem a poluição sonora no ambiente urbano?

\section{Metodologia}

A comparação dos efeitos da pandemia e das medidas para refreá-la com a realidade anterior à pandemia, tal qual propomos em relação à poluição sonora neste trabalho, ainda é precoce. Nem todos os dados necessários para essa comparação estão consolidados, e as conjunturas são dinâmicas e distintas em relação ao tempo e ao espaço - uma mesma cidade pode adotar diferentes medidas restritivas ao longo de um mesmo mês, que, por sua vez, podem ser completamente diferentes de cidades circunvizinhas. Ainda assim, alterações em tendências sociais e comportamentais podem, de fato, ser percebidas pela população e por algumas fontes de registro locais - no caso da poluição sonora, as publicações da imprensa e a divulgação das notificações acerca de poluição sonora feitas às autoridades municipais, por exemplo, podem funcionar como sinalizadores de um contexto maior que, admitidamente, ainda não é possível ser compreendido em sua totalidade.

Por isso, a metodologia aqui utilizada envolveu a pesquisa tanto nas principais bases de dados científicos quanto nos sites agregadores de busca da internet. Nas bases de dados PubMed, Scientific Electronic Library Online (SciELO) e Literatura Latino-Americana e do Caribe em Ciências da Saúde (Lilacs), utilizamos a seguinte estratégia de rastreio: '(noise pollution OR sound pollution) AND (coronavírus OR pandemic)'; abrangendo tais unitermos nos títulos e resumo dos artigos. Complementamos a investigação com artigos adicionais a partir das referências dos trabalhos que encontramos. Já a busca nos sites agregadores Google, Yahoo! e Bing foi feita com os termos 'poluição sonora coronavírus', 'poluição sonora pandemia', 'sound pollution coronavirus' e 'sound pollution pandemic', sem aspas, para encontrar publicações que versassem sobre $o$ assunto. Os resultados das pesquisas foram colhidos até a quinta página de resultados de cada agregador de busca. Posteriormente, tais resultados foram analisados um a um quanto à sua confiabilidade, sendo escolhidos apenas aqueles que consistissem em publicações de órgãos de mídia confiáveis e de órgãos de informação oficiais que mencionassem fontes também confiáveis para seus dados. Adicionalmente, a publicação em si foi analisada para encontrar outras divulgações sobre $\mathrm{o}$ assunto que não foram encontradas pela busca inicial nos agregadores.

\section{Resultados}

\section{Conjuntura paradoxal}

Especialistas indicam que o ruído advindo dos meios de transporte é fundamental para a poluição sonora no contexto urbano, e que é 
esperado sua redução em meio ao isolamento social ${ }^{2}$. Informalmente, cidadãos ao redor do mundo observaram que, com o isolamento social, agora podiam escutar sons que antes eram abafados, como o gorjeio dos pássaros. Erica Walker, pesquisadora em saúde pública da Universidade de Boston, medindo com um decibelímetro o impacto sonoro em sua cidade sob isolamento social, registrou, em uma praça, $68 \mathrm{~dB}$ - antes do isolamento, segundo ela, os registros eram de cerca de $90 \mathrm{~dB}^{3}$. A Associação Brasileira de Qualidade Acústica, ao medir o impacto sonoro em pontos próximos ao Museu de Arte de São Paulo, registrou menos de 10 $\mathrm{dB}$ em locais onde antes do isolamento social se registrava em torno de $70 \mathrm{~dB}^{4}$. $\mathrm{Na}$ Índia Leste, em uma área de intenso ruído devido a pedreiras, o nível sonoro caiu de $85 \mathrm{dBA}$ para menos de $65 \mathrm{dBA}$ após a instituição do isolamento social ${ }^{5}$.

Entretanto, o ruído advindo da vizinhança residencial é, também, uma fonte reconhecida de poluição sonora pela população, que tende a registrar queixas públicas às autoridades quando se sente perturbada ${ }^{6}$. Na cidade de Goiânia, capital do estado de Goiás, a Agência Municipal do Meio Ambiente, responsável pela vigilância ambiental do município, reportou que a quantidade de denúncias de poluição sonora residencial aumentou $199 \%$ entre os meses de março e maio de 2020 - período de vigência de medidas de isolamento social na cidade - em relação ao mesmo período de 2019. A Agência supõe que o aumento se deva ao maior tempo em que as pessoas permanecem em casa, realizando mais confraternizações e festas em horários de sono ${ }^{7}$. Em Birmingham, as queixas de barulho doméstico - aqueles cujas fontes são latidos de cachorros, música, televisão e comportamento antissocial - aumentaram em $82 \%$ entre 23 de março e 6 de maio de 2020 em relação ao ano anterior ${ }^{8}$. Em Tóquio, as queixas por barulho excessivo aumentaram 28,5\% entre março e abril de 2020 em relação ao mesmo período em 2019. Em ocasiões extremas, a perturbação causada pelo barulho de vizinhos levou ao homicídio de um adulto e ao esfaqueamento de um idoso?.

Observamos, portanto, que os dados preliminares indicam uma divergência a respeito da redução ou aumento da poluição sonora em ambiente urbano na vigência das medidas de isolamento social instituídas em resposta à pandemia. Notadamente, entretanto, essa divergência de resultados parece ser fruto de uma divergência metodológica.

Os dados que parecem indicar uma redução da poluição sonora fizeram uso de equipamentos de mensuração do impacto acústico em locais nos quais, usualmente, circulam grande quantidade de pessoas e veículos, e onde se dão atividades ocupacionais produtoras de ruído intenso. Tendo essa circulação e essas atividades sido restritas pelas medidas de isolamento social, é lógico concluir que a poluição sonora advinda dessas fontes iria se reduzir substancialmente.

Por outro lado, os dados que parecem indicar um aumento da poluição sonora advêm majoritariamente dos registros de ocorrências em que a autoridade competente local foi acionada por cidadãos queixando-se de poluição sonora local, especialmente da vizinhança. Isso pode ser explicado pela grande quantidade de pessoas que, antes, estaria fora de casa em suas atividades ocupacionais diárias, mas agora está confinada ao ambiente doméstico. Assim, não só o tempo de permanência nas moradias aumenta, mas também a quantidade de pessoas simultâneas em seu interior. Dado que estão dispensadas de suas atividades matinais, não seria errôneo supor que muitos agora pratiquem mais atividades de lazer no período noturno, algo que, por sua vez, pode ser gerador de ruído e incômodo para seus vizinhos.

\section{Impacto na saúde mental}

A discussão acerca da poluição sonora é relevante em virtude dos impactos que ela pode causar sobre a saúde. O excesso de ruídos pode afetar a qualidade de vida ao causar aborrecimento, raiva, desprazer, exaustão e sintomas relacionados com o estresse. A exposição a 
barulhos, mesmo que de forma aguda, pode estimular o estresse através dos sistemas nervoso autônomo e endócrino, aumentar da pressão arterial, alterar a frequência cardíaca, e causar a liberação de catecolaminas e glicocorticoides ${ }^{10}$. Além disso, a poluição sonora pode afetar a fisiologia do sono e gerar distúrbios com ele relacionados em adultos. Os ruídos a partir de $33 \mathrm{dBA}$ podem promover um despertar indesejado que, por sua vez, pode gerar, em curto prazo, mau humor, sonolência diurna e prejuízo no desempenho cognitivo"1. Não surpreende, portanto, a ocorrência de casos extremos de violência interpessoal conforme relatado anteriormente em Tóquio.

Se em condições habituais a poluição sonora pode produzir esses efeitos, no contexto da pandemia do SARS-CoV-2, eles podem ser ainda maiores. $\mathrm{O}$ impacto tanto da pandemia em si quanto das medidas implementadas contra ela sobre a saúde mental da população vem sendo objeto de interesse de vários estudos recentes. Um deles, por exemplo, afirma que tal contexto pode desencadear uma ampla variedade de problemas psicológicos, como transtorno do pânico, ansiedade e depressão ${ }^{12}$. Os prejuízos sobre a saúde mental que a poluição sonora gera podem, portanto, ser potencializados, bem como funcionar como potencializadores de outros efeitos nocivos.

\section{Considerações finais}

De forma geral, por conseguinte, podemos afirmar que a divergência sobre um aumento ou redução da poluição sonora no ambiente urbano durante o período de isolamento social provocado pela pandemia da Covid-19 depende, em sua maior parte, de qual fonte se considera mais significativa para a produção de poluição sonora. Se as fontes consideradas forem os ruídos advindos de veículos automotivos, de pessoas circulando em vias e praças públicas e de outros equipamentos utilizados em zonas comerciais, então a conclusão, provavelmente, será de redução da poluição sonora nesses ambientes pela medição do impacto sonoro.

Por outro lado, se as fontes consideradas forem os ruídos de mais pessoas em suas próprias residências por mais tempo, de dispositivos sonoros utilizados para lazer e de festas e celebrações que podem se estender noite adentro - já que o isolamento social dispensou muitos de suas obrigações matinais -, então a conclusão, possivelmente, será de aumento da poluição sonora pelas queixas específicas apresentadas pela população às autoridades. Esses dados devem ser levados em consideração em qualquer estudo científico que se proponha a avaliar o assunto de forma mais precisa.

É premente, portanto, o papel de estressor significativo que a poluição sonora pode vir a desempenhar sobre uma população submetida a isolamento social, que se soma a outros vários estressores que, em conjunto, reconhecidamente, prejudicam a saúde mental de pessoas vulneráveis nesse contexto. É fundamental que agentes formuladores de políticas públicas levem esses dados em consideração para traçar e acompanhar suas estratégias de enfrentamento da pandemia.

\section{Colaboradores}
Abe GM (0000-0002-2446-3750)* e Santos LR (0000-0002-5371-0503)* contribuíram igualmente para a elaboração do manuscrito. Teles Filho RV (0000-0003-4822-1526)* con- tribuiu para revisão, formatação e adaptação do manuscrito às normas de publicação. 


\section{Referências}

1. Dunford D, Dale B, Stylianou N, et al. Coronavirus: The world in lockdown in maps and charts [internet]. BBC News. 2020 abr 6. [acesso em 2020 maio 20]. Disponível em: https://www.bbc.com/news/ world-52103747.

2. Ro C. Is Coronavirus Reducing Noise Pollution? [internet]. Forbes. 2020 abr 19. [acesso em 2020 maio 20]. Disponível em: https://www.forbes.com/sites/ christinero/2020/04/19/is-coronavirus-reducing-noise-pollution/\#7e194236766f.

3. Koren M. Four Ways the Coronavirus Is Changing the Planet [internet]. The Atlantic. 2020 abr 19. [acesso em 2020 maio 20]. Disponível em: https://www.theatlantic.com/science/archive/2020/04/coronavirus-pandemic-earth-pollution-noise/609316/.

4. Pereira P, Souza L. Quarentena reduz ruídos urbanos e muda sons da cidade de São Paulo [internet]. Estadão. 2020 abr 11. [acesso em 2020 maio 20]. Disponível em: https://saude.estadao.com.br/noticias/ geral,quarentena-reduz-ruidos-urbanos-e-muda-sons-da-cidade-de-sao-paulo,70003267511.

5. Mandal I, Pal S. COVID-19 pandemic persuaded lockdown effects on environment over stone quarrying and crushing areas. Sci Total Environ [internet]. 2020 [acesso em maio 20]; (732):139281. Disponível em: https://linkinghub.elsevier.com/retrieve/pii/ S0048969720327984.

6. Singh N, Davar SC. Noise Pollution-Sources, Effects and Control. J Hum Ecol [internet]. 2004 [acesso em maio 20]; 16(3):181-7. Disponível em: https://www. tandfonline.com/doi/full/10.1080/09709274.2004. 11905735 .

7. Carneiro M. Queixas de som alto triplicam em Goiânia durante pandemia [internet]. Jornal O Popular. 2020 maio 18. [acesso em 2020 maio 20]. Disponível em: https://www.opopular.com.br/noticias/cidades/ queixas-de-som-alto-triplicam-em-goiânia-durante-pandemia-1.2054838.

8. Cardwell M. Rise in 'noisy neighbour' complaints in Birmingham [internet]. Express \& Star. 2020 maio 21. [acesso em 2020 maio 21]. Disponível em: https://www.expressandstar.com/news/local-hubs/ birmingham/2020/05/21/rise-in-noisy-neighbour-complaints-in-birmingham/.

9. Manichi Japan. Noise complaints on rise in Tokyo as stay-home campaign drags on [internet]. The Manichi. 2020 maio 20. [acesso em 2020 maio 21]. Disponível em: https://mainichi.jp/english/articles/20200520/ p2g/00m/0na/070000c.

10. Basner M, Babisch W, Davis A, et al. Auditory and non-auditory effects of noise on health. Lancet [internet]. 2014 [acesso em 2020 maio 21]; 383(9925):132532. Disponível em: https://linkinghub.elsevier.com/ retrieve/pii/S014067361361613X.

11. Basner M, McGuire S. WHO Environmental Noise Guidelines for the European Region: A Systematic Review on Environmental Noise and Effects on Sleep. Int J Environ Res Public Health [internet]. 2018 [acesso em 2020 maio 21]; 15(3):519. Disponível em: http://www.mdpi.com/1660-4601/15/3/519.

12. Qiu J, Shen B, Zhao M, et al. A nationwide survey of psychological distress among Chinese people in the COVID-19 epidemic: implications and policy recommendations. Gen Psychiatry [internet]. 2020 [acesso em 2020 maio 21]; 33(2):e100213. Disponível em: http://gpsych.bmj.com/lookup/doi/10.1136/gpsych-2020-100213.

Recebido em 27/06/2020

Aprovado em 12/09/2020

Conflito de interesses: inexistente

Suporte financeiro: não houve 